\title{
Efiects of Pressure Sensitivity and Notch Geometry on Notch-Tip Fields
}

\author{
A. AL-ABDULJABBAR and J. PAN \\ Mechanical Engineering and Applied Mechanics \\ The University of Michigan \\ Ann Arbor, Michigan 48109
}

\begin{abstract}
Plane-strain stress and slip-line fields near the sharp and round tips of wedgeshaped notches in perfectly plastic pressure-sensitive materials are investigated to illustrate the effects of pressure sensitivity and notch geometry on notch-tip fields. The Drucker-Prager yield criterion is adopted to describe the material yielding behavior. Notch-tip stress and slip-line fields are first established for a sharp notch. Then the notch-tip stress and slip-line fields for a round notch tip are obtained. The results indicate that for sharp wedge-shaped notches, as the pressure sensitivity increases, the opening stress and hydrostatic tension ahead of the tip decrease. As the wedge angle increases, the opening stress and hydrostatic tension ahead of the tip decrease. For round wedge-shaped notches, as the pressure sensitivity increases, the extent of the exponential spiral zone ahead of the tip increases. As the wedge angle increases, the extent of the exponential spiral zone ahead of the tip decreases and the maximum opening stress and hydrostatic tension ahead of the tip decrease. The closed-form notch-tip stress solutions are useful for design of plastic structural components.
\end{abstract}

\section{INTRODUCTION}

$\mathrm{P}$ ressure-sensitive yielding occurs in polymers [for example, see Sternstein and Ongchin (1), Spitzig and Richmond (2), and Kinloch and Young (3)] and other materials such as cast irons [for example, see Dong et al. (4)], rocks [for example, see Rudnicki and Rice (5)] and soils [for example, see Wu (6)]. To model pressure-sensitive yielding, Drucker and Prager (7) proposed a phenomenological yield criterion that is a linear combination of the effective stress and the hydrostatic stress. The Drucker-Prager yield criterion is a generalization of the Coulomb rule in soil mechanics where the shear stress required for simple slip is linearly dependent upon the normal pressure on the slip surface. The Drucker-Prager yield criterion has successfully been used to describe the plastic behavior of plastics (8) and the slip line patterns around notch tips in plastics under remote tension and compression (9).

Theoretical slip-line solutions based on the pressure-insensitive vor Mises yield criterion were presented for both a sharp crack (10) and a blunted crack (11). The Prandtl solution introduced by Rice (10) for a sharp crack gives no intense straining ahead of the tip. This does not agree with the ductile fracture processes ahead of the tips of mode I cracks observed in experiments. Rice and Johnson (11) introduced the concept of large geometry change of the crack tip and presented a slip-line solution with an exponential spiral zone ahead of a blunted crack tip. The exponential spiral zone gives intense straining ahead of the crack tip.

Recently, some analyses of crack-tip fields have been conducted for pressure-sensitive materials based on the Drucker-Prager yield criterion; for example, see $\mathrm{Li}$ and Pan $(12,13)$ and Dong and Pan (14). Kim and Pan (15) considered the effects of nonsingular stresses on crack-tip fields for pressure-sensitive materials. Jeong et al. (9) studied the plastic deformation and slip-line fields in notched specimens of pure and rubber-toughened epoxies using the Drucker-Prager yield criterion.

It is well known that addition of rubber particles to plastics can significantly increase the fracture toughness of the plastics. Pearson and Yee $(16,17)$ observed that when the rubber-modified epoxies were subjected to loading, the rubber particles in the crack-tip region were cavitated before noticeable plastic deformation of the matrix and a massive shear yielding of the neighboring matrix followed the cavitation of rubber particles. Yee et al. (18) used notched specimens of epoxies in their experiment to investigate toughening of plastics by the addition of rubber particles. The notch in their specimen has a total wedge angle of $45^{\circ}$. They investigated the constraint relief due to the cavitation of rubber particles. Cavitation or void nucleation and growth is intimately related to the macroscopic hydro- 
static tension. Since the notch geometry affects the opening stress and hydrostatic tension ahead of the tip, we here investigate the effects of the wedge angle on the stresses and deformation patterns near a sharp notch tip and a round notch tip in pressure-sensitive materials.

The results of this analysis are used to benchmark the finite element analyses of notched specimens of rubber-modified epoxies modeled as pressure-sensitive porous materials (19) based on the modified Gurson yield criterion introduced by Jeong and Pan (20). Note that when the void volume fraction of the porous materials becomes zero, the modified Gurson yield criterion of Jeong and Pan (20) approaches to the DruckerPrager yield criterion. For porous materials with pressure-sensitive matrices, the macroscopic pressure sensitivity is due to both the intrinsic pressure sensitivity of the matrices and the presence of voids. In fact, Jeong and Pan (21) demonstrated the combined effects of the porosity and the intrinsic material pressure sensitivity on the shear yielding near a crack tip in rubber-modified epoxies.

In this paper, we adopt the Drucker-Prager yield criterion to describe the material yielding behavior. In order to obtain closed-form solutions for engineering applications, the material is assumed to be perfectly plastic. Note that under uniaxial tensile loading conditions, the yield stress remains unchanged for perfectly plastic materials. We first present the stress and slipline solutions for wedge-shaped notches with sharp tips. Then, we present the stress and slip-line solutions for wedge-shaped notches with round tips. Some numerical examples are given and the effects of the pressure sensitivity and the notch geometry on notchtip fields are demonstrated.

\section{STRESS FLED FOR A SEARP NOTCH}

Consider a sharp wedge-shaped notch with a total wedge angle $2 \gamma$ as shown in Fig. 1 where both the Cartesian and polar coordinates are centered at the tip. The stress field near the notch tip can be determined by the results of a general analysis given by Rice (22) for perfectly plastic materials with smooth yield surfaces. Kim and Pan (15) obtained a closed-form

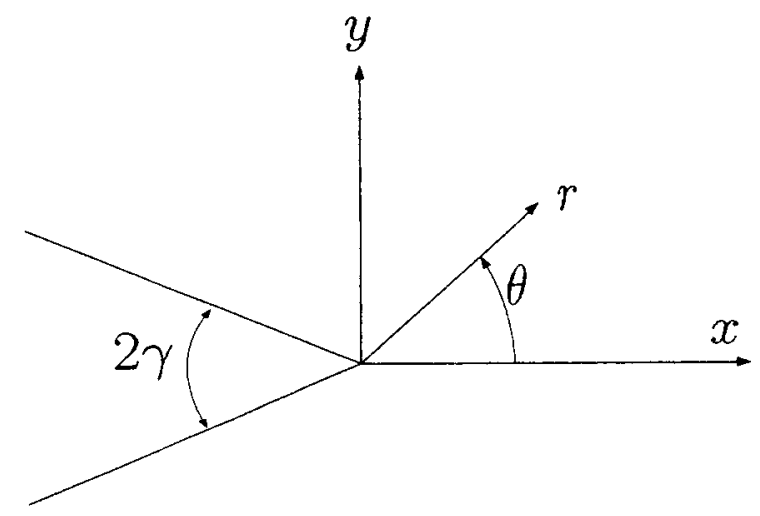

Fig. 1. A sharp notch with a total wedge angle $2 \gamma$. solution for centered fan sectors near a sharp crack tip in pressure-sensitive Drucker-Prager materials. Note that $\mathrm{Li}$ and Pan (12) obtained a closed-form cracktip stress solution for a sharp crack in Drucker-Prager materials using the slip-line theory. Here we develop a closed-form solution for a wedge-shaped notch with $2 \gamma \neq 0$ based on the analysis given by Rice (22).

For pressure-sensitive materials, the Drucker-Prager yield criterion is expressed as

$$
f(\sigma)=\sigma_{e}+\sqrt{3} \mu \sigma_{m}=\sigma_{g e}=\sigma_{0}
$$

where $f(\sigma)$ represents the yield function of the stress tensor $\sigma, \sigma_{e}$ is the effective tensile stress, $\mu$ is the pressure sensitivity factor, $\sigma_{m}$ is the mean stress, and $\sigma_{g e}$ represents the generalized effective tensile stress. For perfectly plastic materials, we take $\sigma_{g e}$ as a constant $\sigma_{0}$. The mean stress is defined as $\sigma_{m}=\sigma_{k k} / 3$, and the effective tensile stress is defined as $\sigma_{e}=\left[3 s_{i j}\right.$ $\left.s_{i j} / 2\right)^{1 / 2}$ where $s_{i j}$ are deviatoric stresses defined as $s_{i j}$ $=\sigma_{i j}-\sigma_{m} \delta_{i j}$. Here, $\sigma_{i j}$ represent the stress components, and $\delta_{i j}$ represents the Kronecker delta. The summation convention is adopted for repeated indices. The indices $i, j$, and $k$ range from 1 to 3 . For polymeric materials, Kinloch and Young (3) reported that $\mu$ ranges from 0.10 to 0.25 . For nodular cast iron, Dong et al. (4) reported that the value of $\mu$ is 0.28 .

In the polar coordinate system, the yield criterion for rigid perfectly plastic materials under plane strain conditions takes the form (12)

$\left(1-\frac{\mu^{2}}{3}\right)^{1 / 2}\left[\left(\frac{\sigma_{\pi}-\sigma_{\theta \theta}}{2}\right)^{2}+\sigma_{r \theta}^{2}\right]^{\frac{1}{2}}+\mu\left(\frac{\sigma_{\pi}+\sigma_{\theta \theta}}{2}\right)=\tau_{0}$

where $\tau_{0}\left(=\sigma_{0} / \sqrt{3}\right)$ is the generalized effective shear stress at yielding. For convenience, we introduce the angle $\phi$ and the parameter $c$ defined by

$$
\begin{aligned}
& \sin \phi=\frac{\mu}{\left(1-\frac{\mu^{2}}{3}\right)^{1 / 2}} \\
& c=\frac{\tau_{0}}{\left(1-\frac{4}{3} \mu^{2}\right)^{1 / 2}}
\end{aligned}
$$

Then, the yield criterion in $E q 2$ can simply be expressed as

$$
\left[\left(\frac{\sigma_{\pi}-\sigma_{\theta \theta}}{2}\right)^{2}+\sigma_{r \theta}^{2}\right]^{\frac{1}{2}}+\sin \phi\left(\frac{\sigma_{\pi}+\sigma_{\theta \theta}}{2}\right)=c \cos \phi
$$

As discussed in Rice (22), there are two types of singular plastic sectors near a sharp crack or notch tip. The first type is referred to as the constant stress sector where the Cartesian stress components are constants in the sector. The second type is the centered fan sector where the radial lines represent a family of characteristic lines of the hyperbolic stress equations. Figure 2 shows a possible assembly of notch-tip sectors surrounding the tip of a sharp notch under fully yielded conditions. Because of the symmetry with respect to the $x$ axis, we consider only the notch-tip sec- 


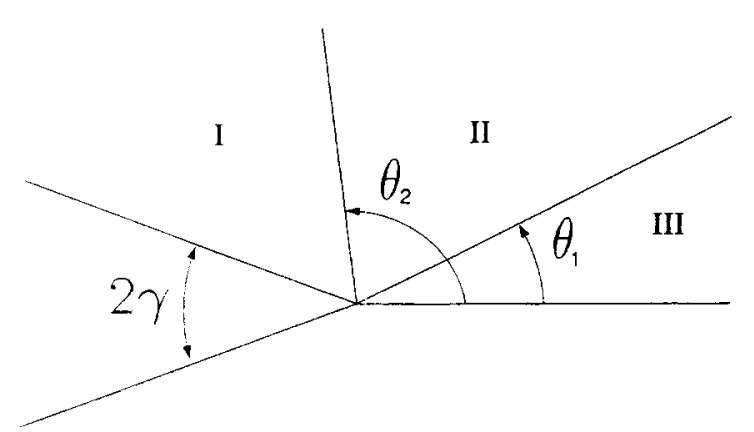

Fig. 2. The assembly of notch-tip sectors near a sharp notch tip.

tors for $\theta \geq 0$. In the Figure, the region adjacent to the free surface of the notch is represented by a constant stress sector, denoted as region I. There is another constant stress sector ahead of the notch tip, denoted as region III. The region between these two constant stress sectors is taken to be a centered fan sector, denoted as region II. In the figure, $\theta_{1}$ represents the angle of the border between regions II and III, and $\theta_{2}$ represents the angle of the border between regions I and II. When $\gamma$ approaches to 0 , the assembly of notch-tip sectors becomes the same as that in $\mathrm{Li}$ and Pan (12), which is the case for the sharp crack.

\section{Constant Stress Sector (Region I)}

Referring to Fig. 2, the stress-free boundary conditions of the notch surface require $\sigma_{\theta \theta}=\sigma_{r \theta}=0$ at $\theta=$ $\pi-\gamma$. The yield criterion in Eq 5 gives $\sigma_{\pi}$ at $\theta=\pi-\gamma$ as

$$
\sigma_{r r}=2 c \frac{\cos \phi}{1+\sin \phi}
$$

Note that although $\mathrm{Eq} 5$ yields two solutions for $\sigma_{r}$, the positive one is chosen in $E q 6$ in order to give a positive opening stress at $\theta=0$. The in-plane stresses in the constant stress sector are expressed by the following equations

$$
\begin{gathered}
\sigma_{r r}(\theta)=C+A \cos 2 \theta+B \sin 2 \theta \\
\sigma_{\theta \theta}(\theta)=C-A \cos 2 \theta-B \sin 2 \theta \\
\sigma_{r \theta}(\theta)=-A \sin 2 \theta+B \cos 2 \theta
\end{gathered}
$$

where $A, B$, and $C$ are constants related to the stresses in the Cartesian coordinates as

$$
\begin{gathered}
A=\frac{1}{2}\left(\sigma_{x x}-\sigma_{y y}\right) \\
B=\sigma_{x y} \\
C==\frac{1}{2}\left(\sigma_{x x}+\sigma_{y y}\right)
\end{gathered}
$$

The constants $A, B$, and $C$ are determined by substituting the expression for $\sigma_{r}$ in Eq 6 and $\sigma_{\theta \theta}=\sigma_{r \theta}=0$ into Eqs 7-9. When $A, B$, and $C$ are determined, the stresses in the constant stress sector (region I) can be expressed as

$$
\begin{gathered}
\sigma_{r \pi}=c \frac{\cos \phi}{1+\sin \phi}[1+\cos 2(\gamma+\theta)] \\
\sigma_{\theta \theta}=c \frac{\cos \phi}{1+\sin \phi}[1-\cos 2(\gamma+\theta)] \\
\sigma_{r \theta}=-c \frac{\cos \phi}{1+\sin \phi} \sin 2(\gamma+\theta)
\end{gathered}
$$

\section{Centered Fan Sector (Region II)}

Slip lines for pressure-sensitive materials are inclined at an angle of $\pi / 4+\phi / 2$ from the major principal stress axis [for example, see Li and Pan (12)]. The major principal stress in region $I$ is in the direction of $\theta=\pi-\gamma$. For the continuity of slip lines, the angle of the border between regions I and II, $\theta_{2}$, can be determined as

$$
\theta_{2}=\pi-\gamma-\left(\frac{\pi}{4}+\frac{\phi}{2}\right)=\frac{3 \pi}{4}-\frac{\phi}{2}-\gamma
$$

Substituting $\theta_{2}$ into Eqs 14 and 15 gives $\sigma_{\theta \theta}$ and $\sigma_{r \theta}$ on the side of the constant stress sector at $\theta=\theta_{2}$ as

$$
\begin{gathered}
\sigma_{\theta \theta}=c \cos \phi \\
\sigma_{r \theta}=c(1-\sin \phi)
\end{gathered}
$$

According to Kim and Pan (15), the closed-form stress solutions for a centered fan sector in pressuresensitive materials are

$$
\begin{gathered}
\sigma_{r \pi}=D-\left(\frac{1}{\tan \phi}+2 \tan \phi\right) \sigma_{r \theta}^{*} \exp (2 \theta \tan \phi) \\
\sigma_{\theta \theta}=D-\frac{1}{\tan \phi} \sigma_{r \theta}^{*} \exp (2 \theta \tan \phi) \\
\sigma_{r \theta}=\sigma_{r \theta}^{*} \exp (2 \theta \tan \phi)
\end{gathered}
$$

where $D$ and $\sigma_{r \theta}^{*}$ are constants that can be determined by the traction continuity condition along the border. With these constants determined at $\theta=\theta_{2}$, the stress solutions for the centered fan sector can be expressed as

$$
\begin{aligned}
\sigma_{\pi} & =\frac{c}{\tan \phi}-c\left(\frac{1}{\tan \phi}+2 \tan \phi\right) \\
(1-\sin \phi) \exp [2 \tan \phi\{(\gamma+\theta)-3 \pi / 4+\phi / 2\}] & (22) \\
\sigma_{\theta \theta} & =\frac{c}{\tan \phi}-c \frac{(1-\sin \phi)}{\tan \phi} \\
& \quad \exp [2 \tan \phi\{(\gamma+\theta)-3 \pi / 4+\phi / 2\}] \\
\sigma_{r \theta} & =c(1-\sin \phi) \exp [2 \tan \phi\{(\gamma+\theta)-3 \pi / 4+\phi / 2\}]
\end{aligned}
$$

\section{Constant Stress Sector (Region III)}

Since the major principal stress of the constant stress sector ahead of the tip (region III) should be in the $y$ direction, the angle of the border between regions II and III, $\theta_{1}$, is determined as 


$$
\theta_{1}=\frac{\pi}{4}-\frac{\phi}{2}
$$

Then, from Eqs 23 and 24, $\sigma_{\theta \theta}$ and $\sigma_{r \theta}$ on the centered fan side at $\theta=\theta_{1}$ are

$$
\begin{gathered}
\sigma_{\theta \theta}=\frac{c}{\tan \phi}-c \frac{(1-\sin \phi)}{\tan \phi} \exp [\tan \phi(2 \gamma-\pi)] \\
\sigma_{r \theta}=c(1-\sin \phi) \exp [\tan \phi(2 \gamma-\pi)]
\end{gathered}
$$

Note that $\sigma_{x y}=0$ ahead of the notch tip due to symmetry. We again resort to Eqs 7-9 for constant stress sectors, along with Eqs 26 and 27 to get the stresses in the Cartesian coordinates in region III as

$$
\begin{gathered}
\sigma_{x x}=c\left[\frac{1}{\tan \phi}-\left(\frac{1}{\tan \phi}+\tan \phi+\frac{1}{\cos \phi}\right)\right. \\
(1-\sin \phi) \exp (\tan \phi(2 \gamma-\pi))] \\
\sigma_{y y}=c\left[\frac{1}{\tan \phi}-\left(\frac{1}{\tan \phi}+\tan \phi-\frac{1}{\cos \phi}\right)\right. \\
(1-\sin \phi) \exp (\tan \phi(2 \gamma-\pi))] \\
\sigma_{x y}=0
\end{gathered}
$$

Expressed in terms of the polar coordinates, the stresses are

$$
\begin{array}{r}
\sigma_{r r}=c\left[\frac{1}{\tan \phi}-\left(\frac{1}{\tan \phi}+\tan \phi+\frac{\cos 2 \theta}{\cos \phi}\right)\right. \\
(1-\sin \phi) \exp (\tan \phi(2 \gamma-\pi))] \\
\sigma_{\theta \theta}=c\left[\frac{1}{\tan \phi}-\left(\frac{1}{\tan \phi}+\tan \phi-\frac{\cos 2 \theta}{\cos \phi}\right)\right. \\
(1-\sin \phi) \exp (\tan \phi(2 \gamma-\pi))] \\
\sigma_{r \theta}=c \frac{\sin 2 \theta}{\cos \phi}(1-\sin \phi) \exp [\tan \phi(2 \gamma-\pi)]
\end{array}
$$

From Eqs 28 and 29 or Eqs 31 and 32, the decreases of $\sigma_{\pi}$ and $\sigma_{\theta \theta}$ ahead of the notch tip at $\theta=0$ when $\gamma$ increases from 0 are

$$
\begin{aligned}
\Delta \sigma_{\pi r}= & \frac{c}{\tan \phi} \exp (-\pi \tan \phi)[\exp (2 \gamma \tan \phi)-1] \\
\Delta \sigma_{\theta \theta}= & \frac{c(1-\sin \phi)^{2}}{\sin \phi \cos \phi} \\
& \exp (-\pi \tan \phi)[\exp (2 \gamma \tan \phi)-1]
\end{aligned}
$$

\section{STRESS FIELD FOR A ROUND NOTCE}

In this section, we consider a wedge-shaped notch with a round tip as shown in Fig. 3 . Note that the origin of the Cartesian and polar coordinates is located at the center of the circular arc of the round tip. The radius of the circular arc of the round tip is denoted as a. For Mises materials, Rice and Johnson (11) showed that directly ahead of the notch tip, the slip lines are exponential spirals and the stress field is the same as

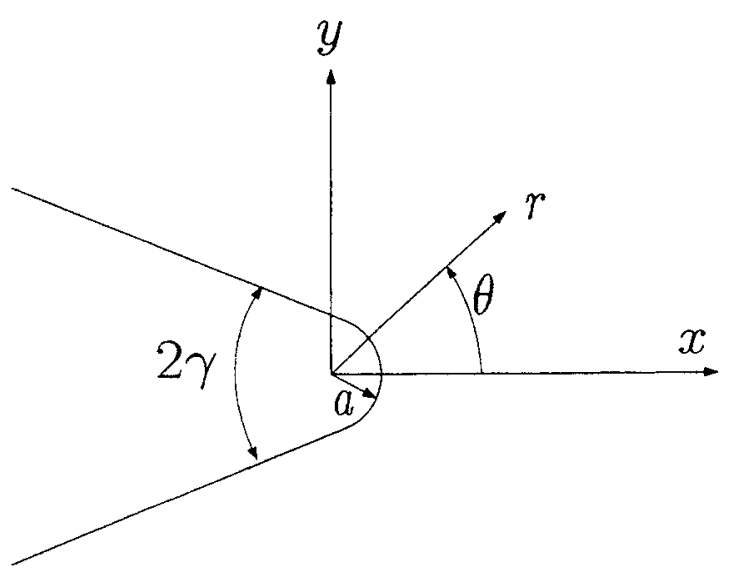

Fig. 3. A round notch tip with a total wedge angle $2 \gamma$.

that for a circular hole under axisymmetric loading; for example, see Kachanov (23).

Jeong et al. (9) gave the slip-line solution for a circular hole in pressure-sensitive materials under axisymmetric loading. The slip lines are represented as

$$
\begin{gathered}
\left(\cos \frac{\phi}{2}+\sin \frac{\phi}{2}\right) \theta-\left(\cos \frac{\phi}{2}-\sin \frac{\phi}{2}\right) \\
\ln \frac{r}{a}=\operatorname{constant}(\text { for } \alpha \text { lines) } \\
\left(\cos \frac{\phi}{2}+\sin \frac{\phi}{2}\right) \theta+\left(\cos \frac{\phi}{2}-\sin \frac{\phi}{2}\right) \\
\ln \frac{r}{a}=\text { constant (for } \beta \text { lines) }
\end{gathered}
$$

The solution ahead of a wedge-shaped notch is similar to the solution ahead of a straight notch except that the extent of the exponential spiral region ahead of the wedge-shaped notch tip is smaller because of the existence of the wedge angle. A schematic plot of the assembly of sectors is presented in Fig. 4. The exponential spiral region ahead of the tip is denoted as region $\mathrm{IV}$ in the figure. As the radial distance from the tip becomes large, the solution should approach to that for a sharp notch tip. Therefore, regions I, II and III are

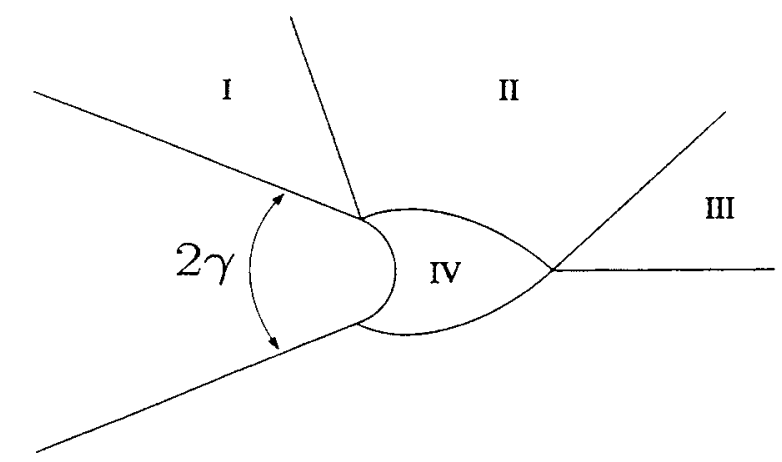

Fig. 4. The assembly of notch-tip sectors near a round notch tip. 
(a)

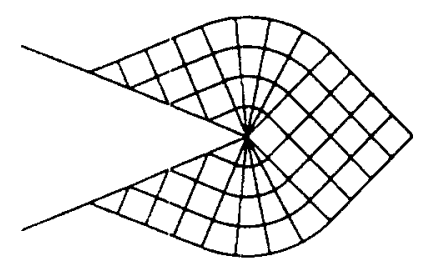

(b)

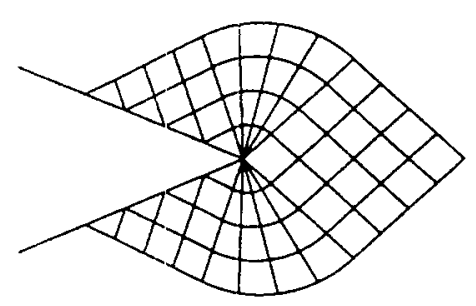

(c)

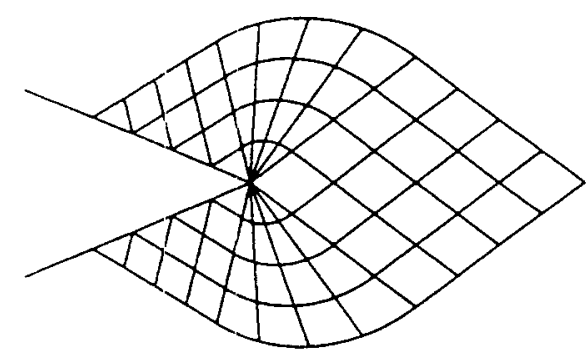

Fig. 5. Notch-tip slip-line fields for $\gamma=\pi / 8$. (a) $\mu=O$, (b) $\mu=$ 0.13 . (c) $\mu=0.3$.

denoted in the figure as those for a sharp notch. The stress field in the exponential spiral region is represented as (9)

$$
\begin{gathered}
\sigma_{r r}=\frac{c}{\tan \phi}\left[1-\left(\frac{r}{a}\right)^{-2 \sin \phi} \frac{\sin \phi}{1+n}\right. \\
\sigma_{\theta 0}=\frac{c}{1+\frac{c}{\sin \phi}}\left[\frac{1-\sin \phi}{\tan \phi}\left\{1-\left(\frac{r}{a}\right)^{\frac{-2 \sin \phi}{1+\sin \phi}}\right\}-2 \cos \phi\right] \\
\sigma_{r i}=0
\end{gathered}
$$

The exponential spiral region is bounded by the $\beta$ lines emanating from the point where the straight wedge surface meets the circular surface of the round tip. The largest extent of the region is located directly ahead of the notch tip at

$$
r=\exp \left[\left(\frac{\pi}{2}-\gamma\right) \frac{\cos \frac{\phi}{2}+\sin \frac{\phi}{2}}{\cos \frac{\phi}{2}-\sin \frac{\phi}{2}}\right]
$$

\section{NUMERTCAC RESULTS}

Based on the solutions developed in the preceding section, the effects of the pressure sensitivity and the (a)

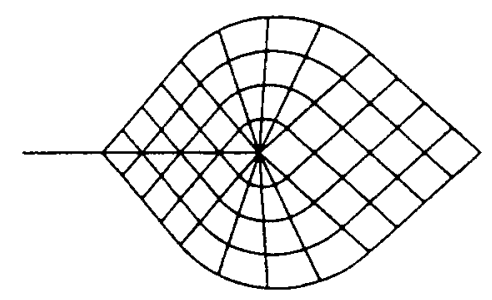

(b)

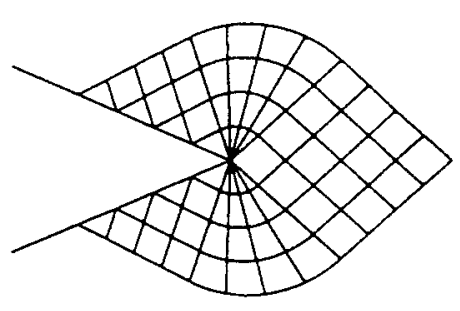

(c)

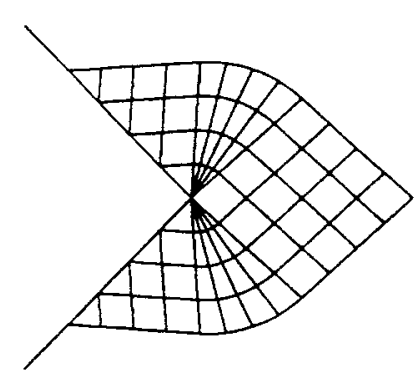

Fig. 6. Notch-tip slip-line fields for $\mu=0.13$. (a) $\gamma=0$, (b) $\gamma=$ $\pi / 8$, (c) $\gamma=\pi / 4$.

notch geometry on notch-tip fields are demonstrated by some numerical examples. Figure 5 shows the slipline fields surrounding sharp wedge-shaped notches with $\gamma=\pi / 8$ for $\mu=0,0.13$ and 0.3 . Here we take $\gamma=$ $\pi / 8$ to represent the wedge angle of the specimens used in Yee et al. (18). We take $\mu=0.13$ to represent the pressure sensitivity of epoxies (9). We also take $\mu=$ 0.3 to represent a material with a larger pressure sensitivity. For example, $\mu$ is 0.28 for nodular cast iron as reported by Dong et al. (4). In Fig. 5, we can see that for pressure-sensitive materials ( $\mu=0.13$ and 0.3 ), the slip lines are not orthogonal to each other as those in Mises materials $(\mu=0)$. For $\mu=0.13$, these slip lines are inclined at $97.5^{\circ}$ and $82.5^{\circ}$ to each other. For $\mu=0.3$, the slip lines are inclined at $107.7^{\circ}$ and $72.3^{\circ}$.

Figure 6 shows the slip lines surrounding a sharp crack tip $(\gamma=0)$ and sharp wedge-shaped notch tips $(\gamma=\pi / 8$ and $\pi / 4)$ for $\mu=0.13$. The effect of the wedge angle is to reduce the angular span of the centered fan sector (region II) by $\gamma$. As $\gamma$ increases, the angular span of the centered fan sector decreases. The angular spans of the two constant stress sectors are not affected. The decrease of the angular span of the centered fan sector results in smaller values of $\sigma_{x x}$ and $\sigma_{y y}$ in the constant stress sector (region III) ahead of the notch tip. 

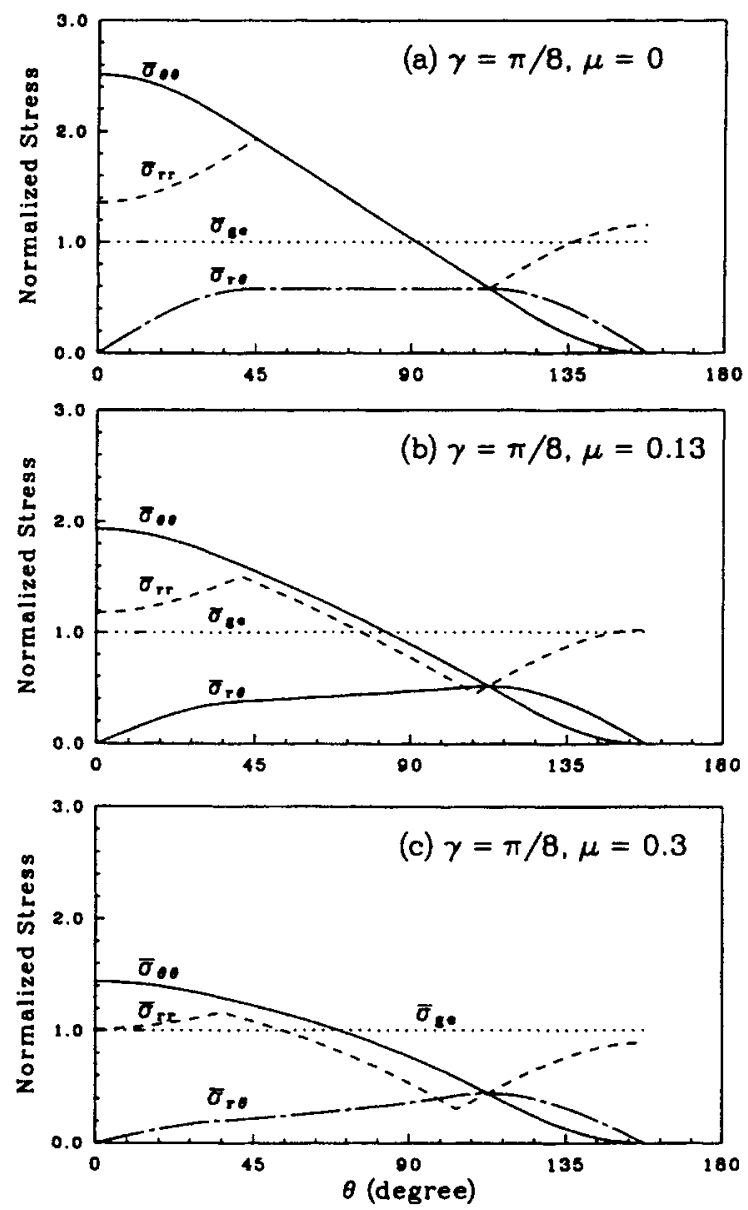

Fig. 7. Angular variations of the notch-tip stresses normalized by $\sigma_{o}$ for a sharp notch with $\gamma=\pi / 8$. (a) $\mu=0$, (b) $\mu=$ $0.13,(c) \mu=0.3$.

The angular variations of the normalized notch-tip stresses $\bar{\sigma}_{i j}\left(=\sigma_{i j} / \sigma_{0}\right)$ for sharp notches with $\gamma=\pi / 8$ are plotted in Fig. 7 for $\mu=0,0.13$ and 0.3. We can see in the figure that $\bar{\sigma}_{\pi}$ and $\bar{\sigma}_{\theta \theta}$ at $\theta=0$ decreases as $\mu$ increases. The angular variations of the normalized notch-tip stresses $\bar{\sigma}_{i j}$ for sharp notches are plotted in Fig. 8 for $\mu=0.13$ and $\gamma=0, \pi / 8$ and $\pi / 4$. We can see in Fig. 8 that at $\theta=0, \bar{\sigma}_{\pi}$ and $\bar{\sigma}_{\theta \theta}$ decreases as $\gamma$ increases.

The variations of the normalized stresses ahead of a sharp notch tip as functions of the wedge angle are shown in Fig. 9 for $\mu=0,0.13$ and 0.3. We can see that as $\gamma$ increases, both $\bar{\sigma}_{r r}$ and $\bar{\sigma}_{\theta \theta}$ decrease. $\bar{\sigma}_{r r}$ and $\bar{\sigma}_{\theta \theta}$ range from their maxima at $\gamma=0$, which represents the sharp crack tip case, to their minima at $\gamma=$ $\pi / 2$, which represents the degenerated case where the notch disappears and $\bar{\sigma}_{\theta \theta}$ represents the normalized uniaxial yield stress under plane strain conditions.

Figure 10 shows the slip lines near round wedgeshaped notches for $\gamma=\pi / 8$ and $\mu=0,0.13$ and 0.3 . We can see that when the pressure sensitivity increases, the extent of the exponential spiral zone ahead of the tip increases. The maximum extents of the exponential spiral regions at $\theta=0$ can be obtained from
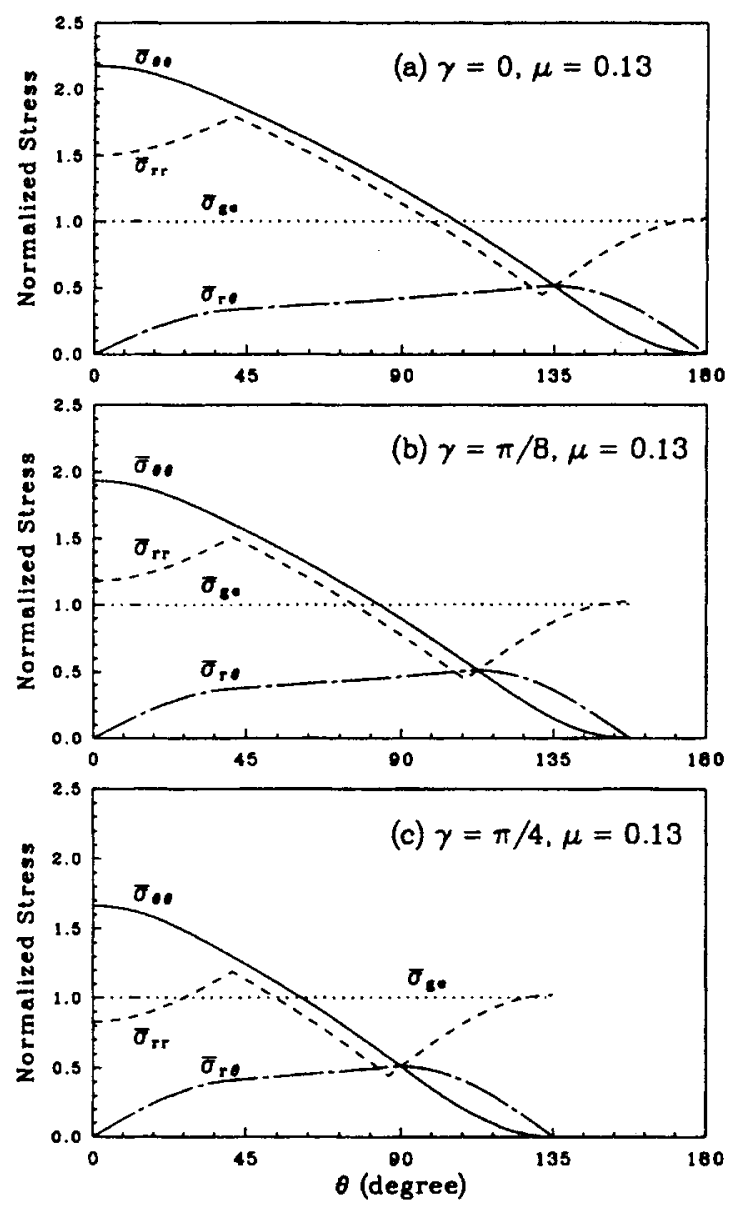

Fig. 8. Angular variations of the notch-tip stresses normalized by $\sigma_{0}$ for a sharp notch with $\mu=0.13$. (a) $\gamma=0$, (b) $\gamma=$ $\pi / 8$, (c) $\gamma=\pi / 4$.

$E q 41$ for $\mu=0,0.13$ and 0.3 as $3.25,3.83$, and 5.02, respectively.

Figure 11 shows the slip lines surrounding round notch tips for $\mu=0.13$ and $\gamma=0, \pi / 8$ and $\pi / 4$. As

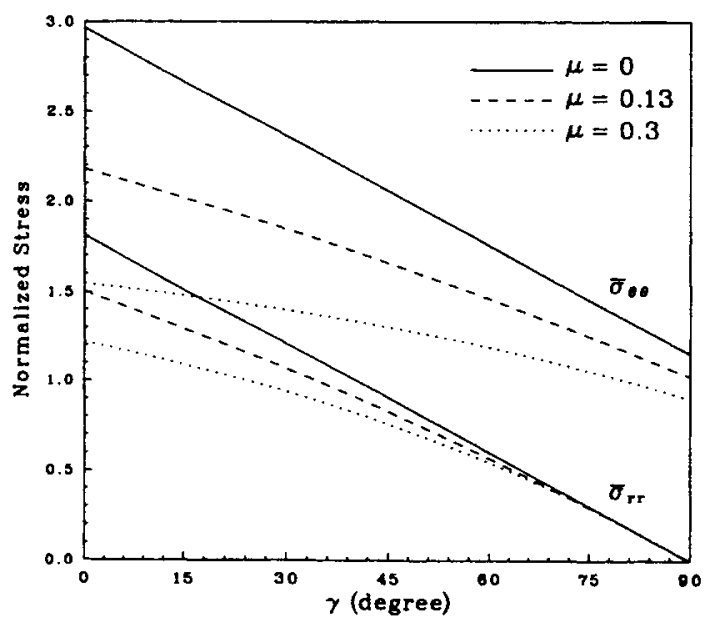

Fig. 9. Variations of notch-tip stresses normalized by $\sigma_{O}$ ahead of a sharp notch tip as functions of the wedge angle $\gamma$ for $\mu=0,0.13$, and 0.3 . 
(a)

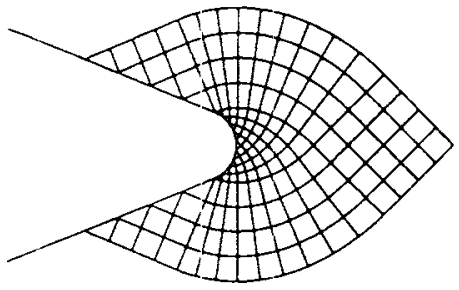

(b)

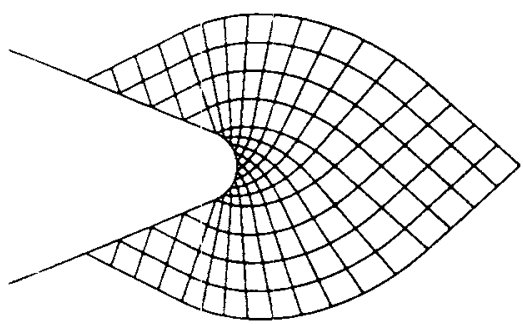

(c)

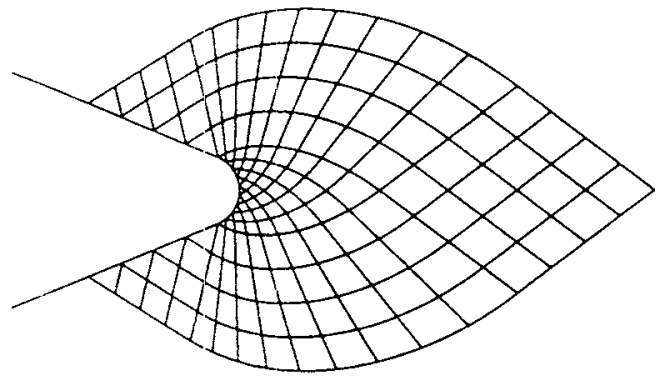

Fig. 10. Slip-line fields near round notch tips for $\gamma=\pi / 8$. (a) $\mu=0,(b) \mu=0.13$, (c) $\mu=0.3$.

shown in the Figure, the extent of the exponential spiral region ahead of the tip decreases as the wedge angle $\gamma$ increases. The maximum extents of the exponential spiral regions at $\theta=0$ can also be obtained from $E q 41$ for $\gamma=0, \pi / 8$ and $\pi / 4$ as $4.81,3.83$, and 2.48 , respectively.

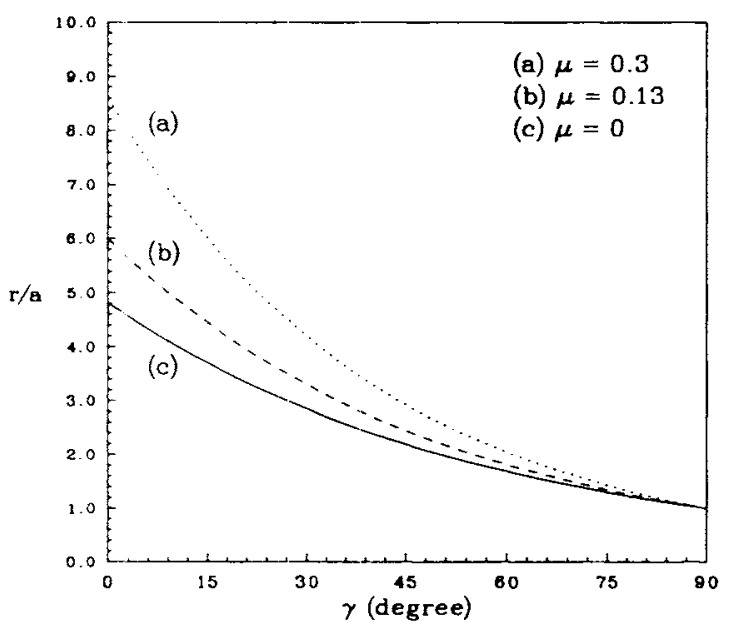

Fig. 12. The maximum extents of the exponential spiral region ahead of a round notch tip as functions of the wedge angle $\gamma$ for $\mu=0,0.13$, and 0.3 . (a)

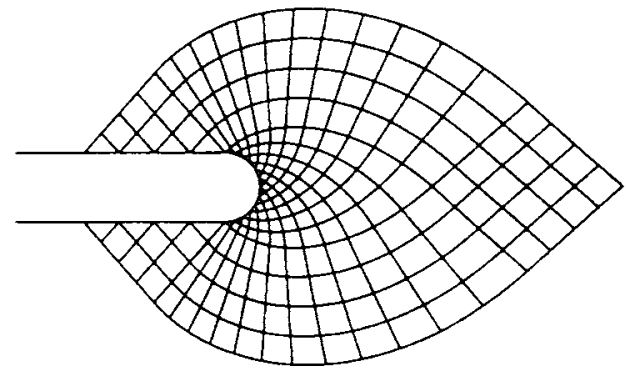

(b)

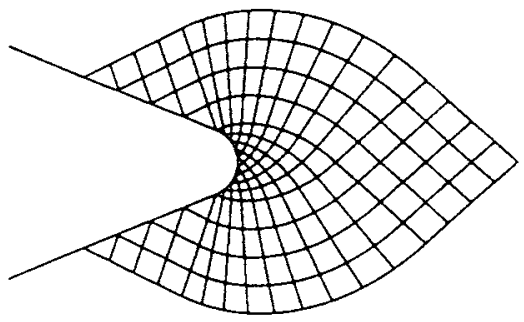

(c)

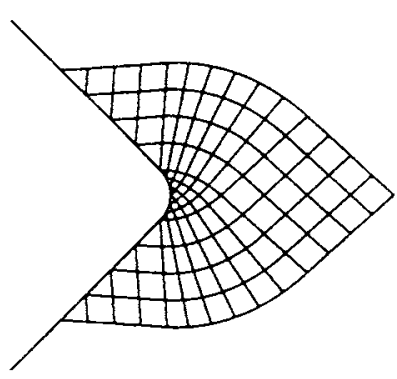

Fig. 11. Slip-line fields near round notch tips for $\mu=0.13$. (a) $\gamma=0$, (b) $\gamma=\pi / 8$, (c) $\gamma=\pi / 4$.

The maximum extents of the exponential spiral zone, $r / a$, as functions of $\gamma$ for $\mu=0,0.13$ and 0.3 are plotted in Fig. 12. As shown in the figure, $r / a$ decreases as $\gamma$ increases. The maximum of $r / a$ occurs at $\gamma=0$. As $\gamma$ reaches $\pi / 2, r / a$ approaches 1 . This is the degenerated case at which the notch disappears and no stress concentration is present.

Figure 13 shows the stresses normalized by $\sigma_{0} \mathrm{di}-$ rectly ahead of a round notch tip as functions of $r / a$ for $\gamma=\pi / 8$ and $\mu=0,0.13$ and 0.3 . We can see that as the pressure sensitivity increases, the extent of the exponential spiral zone increases and the normalized maximum stresses at the maximum extent of the exponential spiral region decrease. The maximum extent of the exponential spiral region is determined at the point where the stresses become constant as $r / a$ increases as shown in Fig. 13.

Figure 14 shows the stresses normalized by $\sigma_{0}$ directly ahead of a round notch tip as functions of $r / a$ for $\mu=0.13$ and $\gamma=0, \pi / 8$ and $\pi / 4$. As shown in the Figure, the effect of the wedge angle is on the extent of the exponential spiral region rather than on the stress distribution within the region. As $y$ increases from 0 


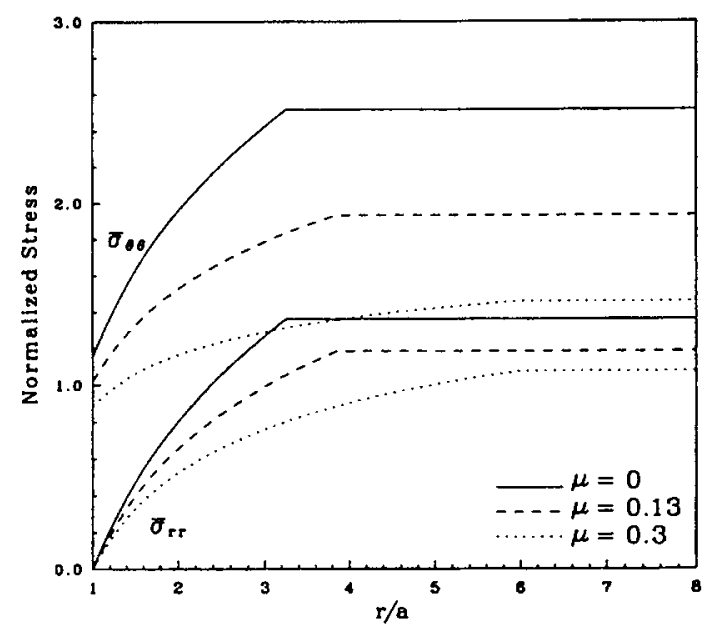

Fig. 13. Notch-tip stresses normalized by $\sigma_{0}$ directly ahead of the tip as functions of $r / a$ for $\gamma=\pi / 8$ and $\mu=0,0.13$, and 0.3 .

to $\pi / 4$, the extent of the exponential spiral zone decreases and, consequently, the maximum values of $\overline{\boldsymbol{\sigma}}_{r}$ and $\bar{\sigma}_{\theta \theta}$ decrease.

\section{CONCLUSIONS}

In this paper, we demonstrate the effects of pressure sensitivity and notch geometry on notch-tip fields in pressure-sensitive materials. We present closed-form solutions for wedge-shaped notches with sharp tips and round tips. The closed-form solutions can be used for further analyses of the cavitation of rubber particles in plastics as for the experiments of Yee et al. (18) on toughening of plastics. Also, the closed-form solutions are useful for design of plastic structural components.

\section{ACKNOWLEDGMENT}

A. A. acknowledges the financial support of the Technical College of Riyadh, Riyadh, Saudi Arabia, and J. P. acknowledges the support of this work by NSF grant under no. DMR-8708405.

\section{REFERENCES}

1. S. S. Sternstein and L. Ongchin, American Chemical Society of Polymers Preprint, 10 (1969).

2. W. A. Spitzig and O. Richmond, Polym. Eng. Sci, 18 , 1129 (1979)

3. A. J. Kinloch and R. J. Young, Fracture Behavior of Polymers, Elsevier Applied Sciences, London (1983).

4. M. J. Dong, C. Piour and D. Francois, "Damage influence on the fracture toughness of nodular cast iron: Part II," in press. Metall. Mater. Trans. (1997).

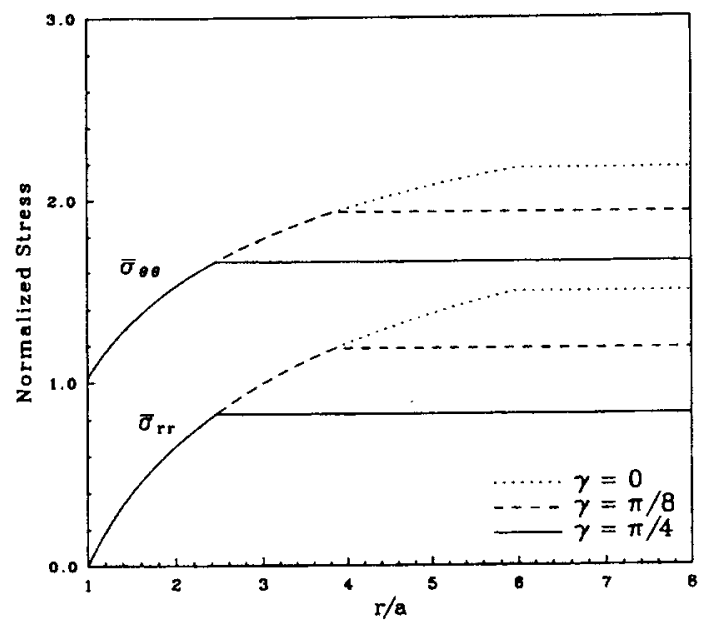

Fig. 14. Notch-tip stresses normalized by $\sigma_{0}$ directly ahead of the tip as functions of $r / a$ for $\mu=0.13$ and $\gamma=0, \pi / 8$, and $\pi / 4$.

5. J. W. Rudnicki and J. R. Rice, J. Mech. Phys. Solids, 23, 371 (1975).

6. T. H. Wu, Soil Mechanics, Allyn and Bacon, Boston (1966).

7. D. C. Drucker and W. Prager, Q. AppL Math, 10, 157 (1952).

8. D. C. Drucker, Metall. Trans., 4, 667 (1973).

9. H.-Y. Jeong, X.-W. Li, A. F. Yee, and J. Pan, Mech. Mater., 18, 29 (1993).

10. J. R. Rice, J. Appl. Mech., 85, 379 (1968).

11. J. R. Rice and M. A. Johnson, Inelastic Behavior of Solids, p. 641, M. F. Kanninen, W. F. Adler, A. R. Rosenfield and R. I. Jaffee, eds., McGraw-Hill, New York (1970).

12. F. Z. Li and J. Pan, J. Appl. Mech. 57, 40 (1990).

13. F. Z. Li and J. Pan, Eng. Fract. Mech., 35, 1105 (1990).

14. P. Dong and J. Pan, Int. J. Solids Struct., 28, 1113 (1991).

15. M. Kim and J. Pan, Int. J. Fract., 68, 1 (1994).

16. R. A. Pearson and A. F. Yee, J. Mater. Sci., 21, 2475 (1986).

17. R. A. Pearson and A. F. Yee, J. Mater. Sci., 26, 3828 (1991).

18. A. F. Yee, D. Li, and X. Li, J. Mater. Sci., 28, 6392 (1993).

19. A. Al-Abduljabbar, $\mathrm{PhD}$ thesis, The University of Michigan, Ann Arbor (1997).

20. H.-Y. Jeong and J. Pan, Int. J. Solids Struct., 32, 3669 (1995).

21. H.-Y. Jeong and J. Pan, Polym. Eng. Sci., 36, 2306 (1996).

22. J. R. Rice, Mechanics of Solids, The R. Hill 60th Anniversary Volume, p. 539, H. G. Hopkins and M. J. Sewell, eds., Pergamon Press, Oxford, England (1982).

23. L. M. Kachanov, Fundamentals of the Theory of Plasticity. Mir Publishers, Moscow (1974). 